\title{
DNA Transformation, Cell Epigenetic Landscape and Open Complex Dynamics in Cancer Development
}

\author{
Naimark O.B..$^{1^{*}}$, Bayandin Yu.V. ${ }^{1 \dagger}$, Beloglazova Yu.A..$^{2 \ddagger}$, Gagarskich O.N. ${ }^{2 \S}$, \\ Grishko V.V. ${ }^{2 * *}$, Nikitiuk A.S. ${ }^{1 \dagger \dagger}$, Voronina A.O. ${ }^{2 * 1}$ \\ ${ }^{1}$ Institute of Continuous Media Mechanics, Ural Branch of RAS, Perm, Russia \\ ${ }^{2}$ Institute of Technical Chemistry, Ural Branch of RAS, Perm, Russia
}

\begin{abstract}
Statistical thermodynamics allowed the formulation of mesoscopic approach of DNA transformation in course of the excitation of collective distortion modes (denaturation bubbles) associated with hydrogen bond breaking between the base pairs. Intermediate (non-continual limit) of DNA modeling (the Peyrard Bishop model) is combined with the field description (generalized Ginzburg Landau approach) to analyze the dynamics of collective open complex modes associated with mesodefects in the DNA ensemble. Collective modes dynamics describes different scenario of gene expression according to statistically predicted form of out-of-equilibrium potential (epigenetic landscape) reflecting specific type criticality of "soft matter" with mesodefects (open complexes) - the structuralscaling transition. Principal difference of thermodynamics of non-continual and continual models is thermalization conditions related to thermal fluctuations responsible for the DNA breathing (localized excitation with breather dynamics) and structural-scaling parameter responsible for spinodal decomposition of out-ofequilibrium potential metastability due to generation of open complex collective modes. Open complex collective modes have the nature of self-similar solutions (breathers, auto-solitary and blow-up modes) of open complex evolution equation accounting qualitative different types of potential metastabilities. Sub-sets of collective modes represent the phase variables of attractors associated with different scenario of expression dynamics, which allows the interpretation of multistability of the epigenetic landscape and the Huang diagram of gene expression. It was shown different epigenetic pathway in attractors phase space corresponding to normal and cancer expression scenario. These scenarios were supported by laser interference microscopy of living normal and cancer cells illustrating multi- and monofractal dynamics.
\end{abstract}

Key words: open complex dynamics, criticality, epigenetics, laser microscopy, normal and cancer cells.

\section{INTRODUCTION}

Cells reveal the structural organization to promptly adapt their mechanobiological environment in realization of fundamental vital cellular functions [1,2]. The mechanobiology properties of living cells are mediated by the cytoskeleton (CSK) realizing also the Gene Regulation Network (GRN) functions [3-6]. The dynamics of the GRN can generate a

\footnotetext{
*naimark@icmm.ru

$\dagger$ buv@icmm.ru

* beloglazova.y@itcras.ru

gagarsckih.olga@yandex.ru

*** grishvic@gmail.com

†nas@icmm.ru

\$voroninaao@gmail.com
} 
multitude persisting states of gene expression as a source of phenotypic variability [7]. A change in network dynamics is caused by the coordinated alteration in the expression status of the genes within the same genome, whereas a change in network structure is caused by a genetic mutation. Nonlinear properties of the network dynamics is related to the CSK epigenetic landscape (a quasi-potential CSK energy) and reflects the influence of the constraints on the realization of expression patterns. Regulatory networks contain non-linear relationships manifested as a multitude attractor states within the network. This coexistence of multiple attractors follows from a quasi-potential multistability [8] that experimentally supported by measuring the convergence of trajectories of genome-wide transcript profile [9]. A coherent development of cell phenotype has the dynamics of the coordinated alteration of the expression modes across the genome towards a more dynamically stable state corresponding to the current constraint. The epigenetic landscape topography represents an 'extended genotype', where the genome determines developmental trajectories and terminal gene expression profiles of cell types and thus, ultimately, the organism phenotype [10].

An attractor transitions represent an intrinsically or externally induced discrete phenotype switch. Thus, attractor states convey a memory allowing the cell to remember a particular gene expression profile induced by previously encountered constraints. It stores information of past perturbations (which can be a random event) but without the need for mutations. The genome and, hence, the topography controls the susceptibility to such perturbation-induced transitions. Beyond the intrinsic interactions in the dynamic network, external control or perturbation of the expression affects the dynamics of the state.

Components of the CSK play a key role in motility, transport and cell division, providing essential scaffolding on which metabolic processes occur. Therefore, cytoskeletal morphology is thought to be a valuable indicator of cell injury and functionality [11]. Inner cytoskeleton structure also provides 'privileged' pathways along which enzymes and substrates are coherently organized and oriented, in order to optimize their interactions [12, 13]. The variety of the mechanisms of structural relaxation could be associated with fundamental property of the cells qualified as the cell plasticity and the cell damage [14].

Plastic deformation as the unique mechanism of the defects induced momentum transfer and the structural memory provides specific CSK organization in mechanobiological environment. The cell plasticity can be considered as the leading mechanism providing the vital CSK properties, including the cell self-organization up to the cell division. The cell division being the vitality ground has also natural links to the defects behavior that provides the evolutionary controlled cell division (due to the preceded plasticity) or the fragility due to the pathological CSK changes leading to the spontaneous cell division. The duality of defects in the realization of the vital cellular functions (plasticity, damage, damage-failure transitions) is stimulating for the consideration of the CSK structure as out-of-equilibrium system with defects taking into account the fundamentals of defects in the matter properties as the localization of the symmetry groups [15]. Multiscale mesoscopic approach in the simulations of biological systems with defects (biological molecules, cell and tissue) are analyzed in the mechanobiology statement to link the qualitative changes of behavior of mesoscopic systems as the specific type of criticality (structural-scaling transitions) [16]. The mesoscopic approach is considered as the "middle-out" paradigm for the description of coherent dynamic behaviors in biological systems and depends upon the choice of leading mechanisms and corresponding mesoscopic thermodynamic (and kinetic) parameters $[17,18]$. The mechanobiology in the combination with molecular genetic approach could provide the actual direction for objectification of pathological tendencies in the living cells in the case of cancer. The methodologies, numerical and experimental techniques coming from mechanobiological approach and combined with multiscale signal processing are the ground of the advanced concepts of biological systems evolution related to the role of collective phenomena [19]. "In-situ" study of the cell mechanobiology by the Laser Interference Microscopy (LIM) with the following definition of "meaningful" 
collective degrees of freedom allows the determination of dynamic stability of biological systems, including tissue, and they qualitative changes with "damage" accumulation with an application to cancer progression.

\section{NON-CONTINUAL (MESOSCOPIC) LIMIT OF GRN MODEL. COLLECTIVE EXCITATION MODES (DENATURATION BUBBLES)}

DNA represents the basic structural biological element (the biological crystal after the Schroedinger definition) and the study of the DNA nonlinear dynamics needs the precise data to establish meaningful thermodynamic properties based on physical experiments [19]. These experiments give data for the DNA dynamics due to the thermal denaturation, i.e. the separation of the two strands by heating, which is also called 'DNA melting' [20, 21]. The denaturation can be monitored experimentally because the breaking of the base pairs is accompanied by a large increase in the absorbance of UV light in the course of sharp transition between the double helix and the separated strands. This transformation has typical features of phase transformation with structural variables (order parameters) associated with the local opening called 'denaturation bubbles'. The specificity of DNA lies in its ability to store genetic information, and the large amplitude highly nonlinear motions providing the reading of this information. The model that encodes the information in DNA is related to the scale of base pair and was proposed by Peyrard and Bishop (PB-model [20]). This model has intermediate mesoscopic nature and reflects the structure of biological molecules as the soft matter combining solid and liquid properties in the Hamiltonian form. DNA undergoes large conformational changes during transcription (i.e. the reading of the genetic code) or replication. The validity of the PB-model was supported by the calibration of parameters and comparison with well-controlled experiments [22]. The ability of the PB-model to describe the DNA dynamics was illustrated by the numerical simulation of the DNA melting as the phase transition induced by numerous collective excitation of the denaturation bubbles in the presence of thermal fluctuations [23, 24]. The thermalized PB-model revealed the existence of localized large amplitude motions appearing as a temporary breaking of energy equipartition in the system separated by narrower 'cold' regions. The nonlinear energy localization corresponds to the area of large amplitude collective motion of the base pairs in the discrete DNA lattice with the so-called breather dynamics appearing as a good explanation for the 'breathing' of the thermalized DNA. Statistical thermodynamics of the breather ensemble revealed the tendency of the free energy release, when nonlinear stacking leads to "self-amplification process". The PB-model of the discrete thermalized DNA lattice reflects the important properties of the Gene Regulation Network to excite the collective modes (denaturation bubbles) for the bridging of the DNA lattice transformation and the field description of the DNA ensemble and the cell dynamics.

\section{OPEN COMPLEX DYNAMICS. DNA AND CELL TRANSFORMATIONS}

The open states or the bubbles appear as the areas of local denaturation due to the breaking of the H-bonds, which leads to the local opening of the base pairs (Fig. 1). The open complexes develop due to the activation of specific subsets of gene expression patterns revealing the intrinsic stochasticity in gene expression, wide range of gene patterns providing different possible phenotypes [17]. The open states provide the DNA transformation as the mechanisms of the cell plasticity and the cell division.

Conformation transitions, the formation of opening states in the processes of the DNAprotein recognition are the consequence of the large-amplitude motions leading to the expression scenario [19]. Mesoscopic description of open complexes assumes the definition of internal "mesoscopic" variables reflecting the coherent behavior of the "minimal molecular set" of the breaking of the H-bonds. This set is close to 50 genes linked to the level of perturbation needed to trigger a multiscale spatial-temporal transition in the system [25, 26]. The ensemble of "minimal set", associated with the open complexes, are responsible for the "structural 
memory" and realization of the cell plasticity, the cell damage, pattern formation, morphology and the shape fractality [16]. The open complexes as a typical mesoscopic defects are associated with the local unwinding, opening of the double helix. Biological processes involve ensembled molecular components (open complexes) responsible for mesoscopic properties, spatialtemporal multiscale organization according to epigenetic (thermodynamic) landscape in which system's transitions are realized [27, 28].

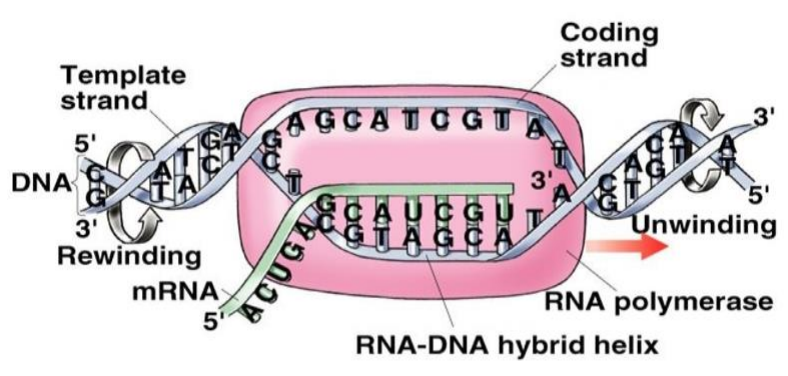

Fig. 1. DNA structure and open complex formation.

Thermodynamic aspects of the open states can be considered as the key problem for the understanding of the DNA functioning. Statistical approach to describe the collective behavior of the DNA distortion modes allowed the formulation of thermodynamics of the open complexes and the evolution equation in the generalized Ginzburg - Landau form reflecting specific type of criticality named as the structural-scaling transition [15]. Structural parameters associated with open complexes and corresponding localized untwisting modes were introduced as the localization of the symmetry group of distortion tensor. The microscopic parameter, that is associated with the open complex volume, represents the local distortion spreading on the small segment $2 \mathrm{~d}$ (about 50 base pairs) with the opening normal distortion mode to the segment area with orientation .The mean value of the open complex for uni-axial case corresponds to the minimum of the out-of-equilibrium free energy in the Ginzburg - Landau form

$$
F=\frac{1}{2} A\left(1-\frac{\delta}{\delta_{*}}\right) p^{2}-\frac{1}{4} B p^{4}+\frac{1}{6} C\left(1-\frac{\delta}{\delta_{c}}\right) p^{6}-D \sigma p+\chi\left(\nabla_{l} p\right)^{2}
$$

$\sigma$ is the external constraint. The value of $\delta$ is the second structural parameter and represents the ratio of two characteristic scales $\delta=(d / l)^{3}$ the length $d(d \sim 30-50 \mathrm{~nm})$ of the DNA segment of open complex nucleation and the distance $l$ between open complexes. The bifurcation points $\delta_{*}=1.3, \delta_{c}=1$ separate qualitative different areas of the free energy non-linearity: transition from the uni-modal to the bimodal form at $\delta_{*}=1.3$ with qualitative changes of the metastability at $\delta_{c}=1$ from the finite to the infinite depth of the second minima). The $\delta$-parameter and the critical values $\delta_{*}=1.3, \delta_{c}=1$ play the role that is similar to the characteristic temperatures in the Ginzburg-Landau theory. The appearance of the $\delta$ - parameter in statisticalthermodynamic model of condensed matter with mesodefects has deep physical meaning as the generalization way of thermodynamic approach for out-of-equilibrium states proposed by Leontovich [29]. The generalization follows to the classical Boltzmann-Gibbs statistics to introduce the effective field and the current thermalization condition for the out-of-equilibrium states. It was shown in [15] that the structural-scaling parameter $\delta$ has the unique property to provide both conditions (correction of the effective field and current thermalization) for out-ofequilibrium mesoscopic systems. The gradient term in (4) describes the non-local interaction in the distortion field; $A, B, C, D$ and $\chi$ are the phenomenological parameters. 


\section{THE GINZBURG - LANDAU FREE ENERGY AS THE EPIGENETIC LANDSCAPE}

The state space of the cells is related to the 'epigenetic landscape' due to 'causal interactions between genes and their products, which bring the phenotype into being' [17, 28, 30, 31]. An epigenetic landscape can be interpreted as a free energy profile based on the entire ensemble of simultaneous interactions having the number for simple organism the order of $10^{7200}$, that could be reconstructed to introduce on the scale of DNA the meaningful mesoscopic variables. The idea of an 'epigenetic landscape' was proposed by Waddington [32] as the sequence of the valleys with the free energy minima in terms of the variables reflecting the coherent gene-bygene behavior. These variables can significantly affect nonlinear processes, thus switching cells between distinct phenotypes, by analogy with phase transition in physical systems. Collective behavior of these variables (collective modes) could provide the reprogramming of cell states that could not be realized by interactions between genes due very high kinetic barriers of the epigenetic landscape $[33,34]$. The energy profiles could be considered in the framework of the Ginzburg - Landau free energy using the internal variables reflecting the coherent gene-bygene behavior as the expression unit related to the open complex. The flatness of epigenetic landscape under transition from unimodal to bimodal energy profile is the sign of the critical point, where the sharp increase of the relaxation time corresponds to the development of a global phase transition in the whole gene expression profile (the mRNA expression [27]) with the shift in the frequency profiles to the coherent mRNA expression modes. According to these results, the critical dynamics of gene expression shows the singularity induced scaling that is characteristic for out-of-equilibrium critical systems. The open complexes and the mRNA coherent expression modes appear due to the decomposition of the free energy metastability. This metastability has different nature for corresponding ranges of the "governing" structuralscaling parameter transforming the unimodal potentials into bimodal potentials with finite and infinite depth depending on the $\delta$-range [16]. The conception of epigenetic landscape provides important conceptual implications for evolutionary biology transforming the GRN mapping into quasi-potential landscape and sheds the nonlinear scenario of the genotype-phenotype transition in the terms of meaningful collective variables of gene expression. It is important that the landscape is not a true energy potential as in classical physics, which gives rise to 'path independence' It arises from the mathematical analysis of the global stability of states of the GRN treated as a dynamic system with rugged sensitivity to the network perturbations. The landscape concept affords general explanatory principles for evolutionary biology if the entire GRN architecture is known [35]. The mutation will alter the landscape, either by inflicting a graduated change, such as distorting the contours of the epigenetic (quasi-potential) valley, altering the height of separating hills (modulating accessibility), or, more dramatically, by destroying of natural transitions along the attractors states [36]. When a new attractor suddenly becomes accessible to follow a mutational landscape distortion (that is generally a rather rare event), it leads to previously inaccessible, gene expression configuration and a new cell type and evolutionary progress could be triggered.

\section{GLOBALLY CONVERGENT DYNAMICS OF GENE EXPRESSION AS AN ATTRACTOR STATES}

Temporal development in gene-expression collective modes was studied to analyze the expression groups sorted according to normalized root-mean-square-fluctuation (Nmsf) and related to an early response (the first $30 \mathrm{~min}$ ) to growth factors in a MCF-7 breast cancer cell population [34,35]. The averaged values of Nrmsf can be considered quantitative as relationship with the mRNA expression. The hill-like distribution function marks a dynamical stable profile of expression that in turn is defined as coherent expression state for a set of genes. As the consequence, it was assumed that the nrmsf can be considered as the order parameter for gene expressions stems with consolidated gene expression scales. It was noted [34] that the 
$n r m s f$ should be related to the physical plasticity of genomic DNA: a higher $n r m s f$ should be associated with a more pliable DNA structure. Nrmsf, as the spatial/temporal variance, should correspond to the degree of fluctuation/freedom in statistical thermodynamics. The log-normal law and the power law that are characteristic for the critical scenario, could link chromatin aggregation and gene expression as coordinated transitional behaviors at the chromosome level with coarse grain dynamics. Kinetics for the open complex parameter can be presented by the evolution equations for mentioned structural variables [16]:

$$
\frac{d p}{d t}=-\Gamma_{p} \frac{\partial F}{\partial p}, \frac{d \delta}{d t}=-\Gamma_{\delta} \frac{\partial F}{\partial \delta},
$$

where $\Gamma_{p}$ and $\Gamma_{\delta}$ are the kinetic coefficients. The path of the bifurcation point $\delta$ leads to penetration into the metastability area and the generation of the collective finite-amplitude distortion modes with breather $\left(\delta \rightarrow \delta_{*}\right)$ and solitary wave $\left(\delta_{*} \rightarrow \delta \rightarrow \delta_{c}\right)$ dynamics (Fig. 2). The solitary wave solution has the form $p(\zeta=p(x-V t))$, where the wave amplitude, velocity and the width of the wave front are given by the self-similar solution:

$$
p=\frac{1}{2} p_{a}\left[1-\tanh \xi L_{B}\right], L_{B}=\frac{4}{p_{a}}\left(2 \frac{\chi}{A}\right)^{1 / 2}
$$

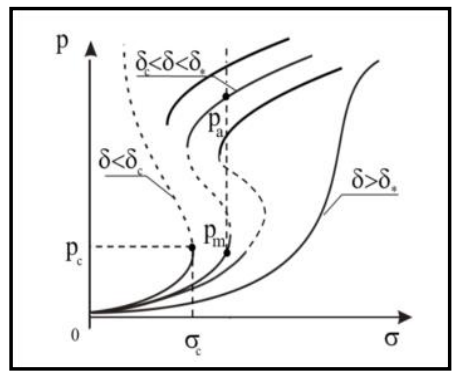

a)

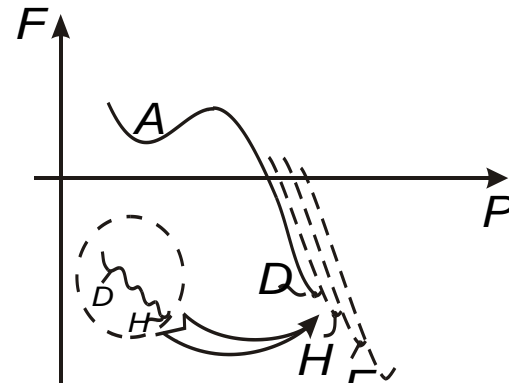

b)

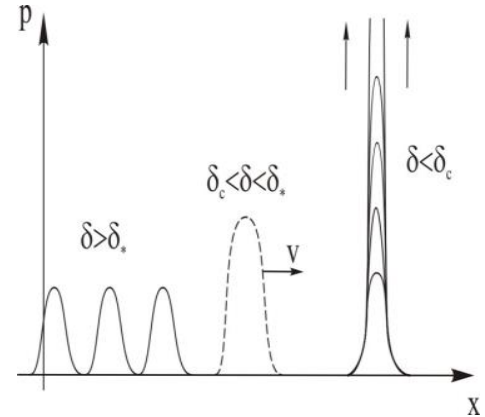

c)

Fig. 2. a) Phase diagram of open complex parameter $p$ versus external constraint $\sigma$; b) Free energy "epigenetic" landscape; c) Self-similar solutions corresponding to breathers $\left(\delta_{*}<\delta\right)$, solitary wave $\left(\delta_{*}>\delta>\delta_{c}\right)$, blow-up $\left(\delta<\delta_{c}\right)$ attractors.

The velocity of solitary wave is $V=\chi A\left(p_{a}-p_{m}\right) / 2 \zeta^{2}$, where $\left(p_{a}-p_{m}\right)$ is the $p$-jump in the metastability area. A transition through the bifurcation point $\delta_{c}$ leads to the qualitative change of the double-wall potential into the form with infinite second minimum depth. These qualitative changes in the metastable potential lead to specific open complex dynamics, generation of collective distortion modes with «blow-up» kinetics [15].

Specific type of self-similar solution determines the kinetics of distortion modes for $t \rightarrow t_{c}$ on the set of spatial scales $L_{H}=k L_{c}, k=1,2, \ldots, K$ :

$$
p(x, t)=\varphi(t) f(\zeta), \zeta=\frac{x}{L_{c}}, \varphi(t)=\Phi_{0}\left(1-\frac{t}{t_{c}}\right)^{-m},
$$

where $m>0, \Phi_{0}>0$ are the parameters related to the nonlinearity of free energy release in metastability, $L_{c}$ and $t_{c}$ are spatial and temporal parameters of "blow-up" self-similar solution. The existence of the set of collective modes and consequent transformation of these modes according to the $\delta$ - kinetics from the breather to solitary and blow-up dynamics illustrates the globally convergent open complex dynamics in the presence of three different attractors (Fig. 2). 
The value of structural-scaling parameter reflects the current susceptibility of the DNA ensemble to the nucleation and growth of the open complexes in the presence of the DNA constraint and the thermal bath. The kinetics of structural-scaling parameter provides the scenario of the spinodal decomposition in different areas of metastability due to the initiation of the open complex collective modes. These modes represent three types of the self-similar solutions of the evolution equations (2) for the open complex parameter $p$ in characteristic areas of structural-scaling parameter: breathers, autosolitary waves and blow-up dissipative structures. The phase spaces of these attractors are linked to the sets of mentioned collective modes. The sets of collective open complex modes (breathers, solitary and blow-up modes), that could co-exist generally in the DNA double helix structure, represent the collective variables subject the non-linear dynamics of out-of-equilibrium biological system to a few preferred global states. The solutions (3), (4) have the nature of the self-similar intermediate asymptotic that allows the consideration of mentioned collective modes as the eigen-function spectrum of nonlinear problems $[15,37,38]$ that explains the gene expression as the resonance pattern forming without modification of the DNA's sequences [39]. The "evolution arrow" follows from the kinetics of structural-scaling parameter $\delta$ that realizes the natural tendency for transformation of breather and solitary modes into the blow-up modes. The interpretation of biological regulation within the framework of nonlinear dynamics of open complexes can be linked to the driving factor of the $\delta$ - kinetics providing the evolution pathway though the areas of different attractors to realize the gene expression scenario and the cell evolution [40]. This analysis demonstrates temporal development of gene-expression as global phase transition $[41,42]$. The robust organization in cells, when the expression mechanism of thousands of genes are coordinated by a few key transcription factors can be linked to attractor states in the gene-expression landscape $[43,44]$ providing the 'phenotypic states'. The attractor concept envisages the system as evolving toward a preferred (minimal energy) state due to appearance of 'globally convergent' solutions [45-48]. These solutions attract the system dynamics in the presence of stochastic fluctuations related to a gene-by-gene interaction. Attractor states are realized in the presence of a rugged non-equilibrium free energy landscape in the terms of mentioned variables as the generalization of the Landau theory of the phase transitions $[49,50]$.

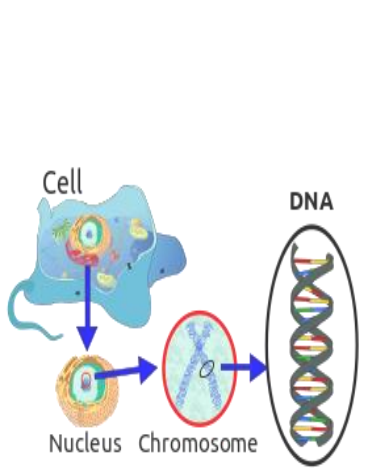

a)

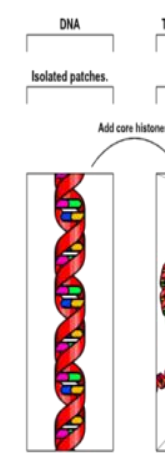

b)

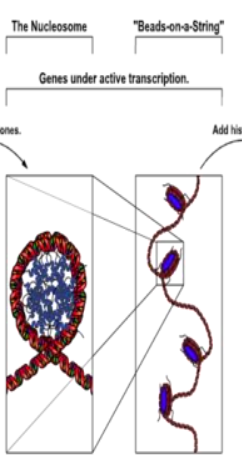

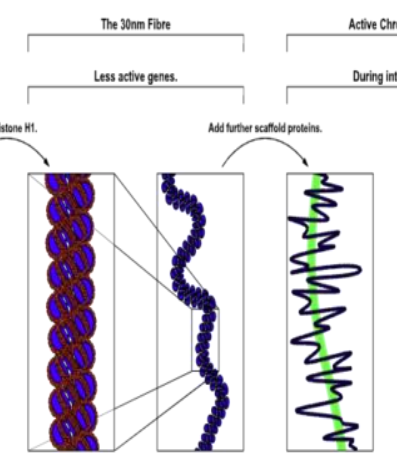

c)

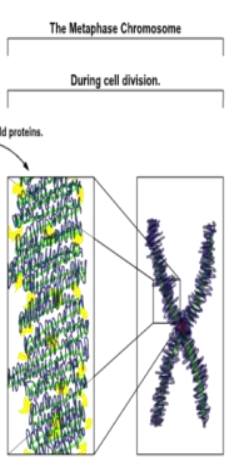

d)

Fig. 3. Cell structure and DNA transformation: a) cell structure, b) histone topology, c) histone package, d) cell division [https:en.wikipedia.org/wiki/Chromatin\#/media/File:Chromatin_Structures.png].

The generalization of the Landau theory for thermodynamics of the DNA ensemble was developed in [16] using the "effective field" approach by Leontovich [29] for the statistical thermodynamics of the out-of-equilibrium "slow driven systems". In the presence of the free energy metastability (1) the $\delta$ kinetics plays the role of the "intelligent agents" (Maxwell's demons) that used to drive the system due to the metastability decomposition [51]. This scenario could clarify a fundamental question concerning the problem of cell dynamics controlling genome-wide expression - What is the 'driving force' that attracts the entire system toward a few preferred global states, thus making the genome act as a single integrated system? [52, 53]. 
The sequence of generation of these modes can be used for the explanation of the DNA transformation associated with the cell plasticity and the cell division (Fig. 3) [54]. The front of autosolitary wave mode has the sharp curvature $\chi=(\partial \mathrm{p} / \partial \mathrm{s})_{f}(s$ is the longitudinal DNA axis) separating the portions of DNA strands with breather and autosolitary wave dynamics that can be associated naturally with transition to the histon topology of the DNA strand (Fig. 3,a) in the condition of continuously increasing $s$-curvature under $\delta \rightarrow \delta_{c}$. The solitary wave dynamics of the open complexes provides the active transcription action caused by the DNA plasticity and the meeting point (centromere) of two strands with inverse curvature can be considered as analogue of "sitting dislocation" with the high-energy barrier. Double helix becomes more condensed due to the histone topology dynamics. Numerous scenario of the histone topology lead to the DNA package ("histone lattice", Fig. 3,b and 3,c) with less active genes and new correlation in the open complex ensemble providing the path of the $\delta_{c}$ critical point. It leads to the excitation of blow-up open complex dynamics inside of histone topology, rupture of the DNA strands and the formation of small $p$-chromosome arms (Fig. 3,d) as the precursor of the cell division [55]. The chromosome consists of the condensed structure of the DNA double helix (10, 000 times than in the normal DNA double-strand). The compact form of chromosomes has four arm structure as a pair of sister strands attached by each other at the centromere. The long $q$-chromosome arms appear due to the tripping of some labile histone stitching (centromere) with low energy barrier after the rupture of the DNA strands in histones. Finally, due to this act of replication a chromosome consists of two sister chromatids. The DNA transformation occurs in order for the proper separation of the genetic material between daughter cells under the cell cycle (Fig. 3,d). Similar scenario was discussed in [35] in early response to growth factors in a MCF-7 breast cancer cell population to characterize the distinct expression domains: static, transit and dynamic domains according to the degree of temporal variation in expression.

\section{SELF-SIMILAR SOLUTIONS AS AN ATTRACTOR STATES. INTERPRETATION OF THE HUANG DIAGRAM}

The dimensions of the gene expression state space can be assimilated according to the Waddington conception as the dimensions of corresponding attractors with dynamics that follows to the sets of self-similar solutions providing the transitions between metastable states realizing the non-genetic mechanisms of temporal selections of epigenetically encoded phenotype. The quasi-potential landscape is a mathematical entity related to molecular (genetic) basis and must be considered as intermediate level in the genotype-to-phenotype correspondence. Conventionally, the self-similarity (scaling) implies similarity based on the invariance solutions at different times that have mathematically the nature of asymptotic solutions reflecting a certain type of stabilization of nonlinear processes under widely varying conditions [56]. A typical example of self-similarity is the formation of solitary waves (solitons) propagating with amplitude-dependent velocities. In a more general sense, the scaling refers to the existence of self-similar solutions with dimensionless variables tending to zero or infinity and providing for the evolution function the finite limit or the power asymptotic form. These classes are classified as self-similar solutions of the first and second kind, respectively [57]. The second type of solutions cannot be derived from dimensional analysis, including estimation of self-similar variables, and the self-similarity is related to a nonlinear eigenvalue problem. 
A)

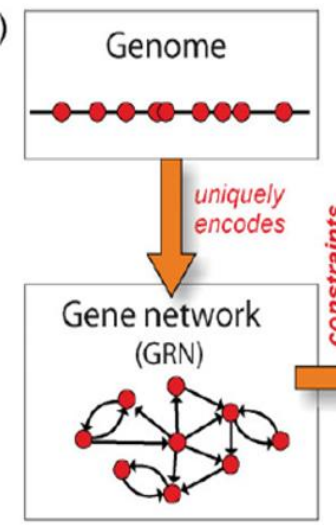

B)

Gene expression pattern $=$ network state

$S\left(t_{1}\right)=\left[x_{1}, x_{2}, x_{51}, x_{8}, x_{51}, x_{0}, x_{7}, x_{8}, x_{5}\right]$
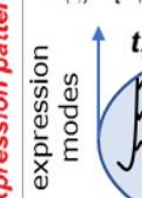

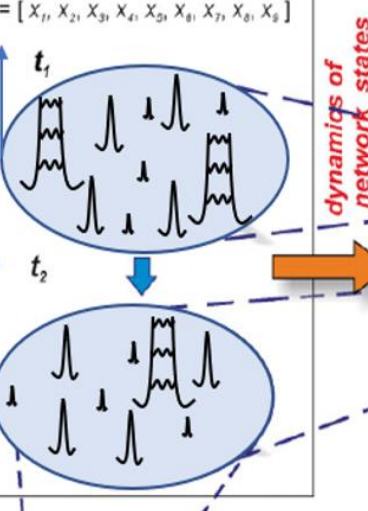

C)
State space (2D)
all possible gene expression patterns $S$ projected to a YX plane . the distance reflects similarity of patterns

D)

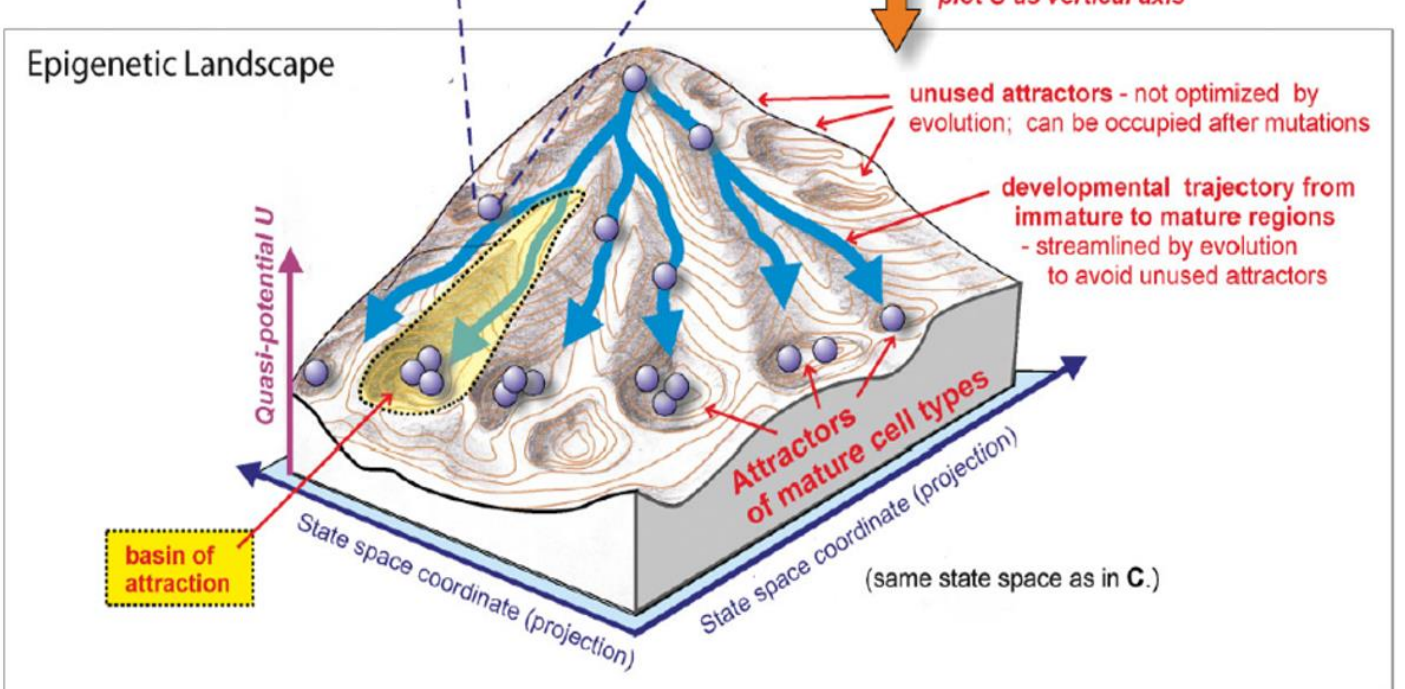

Fig. 4. Modified Huang's diagram of the expression pattern evolution [10].

Of importance for mathematical description and self-similar solutions is to reflect physical mechanisms responsible for localized instability and their multiscale interaction as factors that make the behavior of a system subordinate to self-similar variables and self-similar solutions. The formation of localized instabilities is considered as an indication that the behavior of an out-of-equilibrium system reaches a critical level and is associated, by analogy with critical phenomena, with independent dynamic variables, which obey the spectrum of interacting collective modes, since the initial non-self-similar problem is degenerated.

The breather, autosolitary and blow-up modes represent the sub-sets of dynamic coordinates of different attractors existing in the corresponding ranges of the structural-scaling parameter. The transition along these sub-sets according to the $\delta$ - kinetics provides the natural expression arrow of the cell dynamics (Fig. 3). These results allow the interpretation of the Huang diagram [10], Fig. 4, where the state space $S\left(x_{i}\right)$ is associated with the set of collective modes illustrating the expression dynamics for the given genome and the Gene Regulation Network (Fig. 4,A). Gene expression pattern (Fig. 4,B) represents the network state with the set of coordinates corresponding to the collective (expression) modes providing the constraints modification (rescaling in the term of the $\delta$ - parameter) and the consecutive DNA transformation according to scenario in Fig. 3. The transition between the states $S\left(t_{1}\right), S\left(t_{2}\right)$ corresponds to the natural cell cycle with the cell division at state $S\left(t_{3}\right)$ due to the development of the single blow-up expression mode (Fig. 4,C). 


\section{LASER INTERFERENCE MICROSCOPY OF CELL DYNAMICS. DUCTILE- BRITTLE TRANSITION IN CSK STRUCTURE AS CANCER PRECURSOR}

Open complexes as mesoscopic defects are responsible for two mechanisms in condensed matter associated with plasticity and damage-failure transition [58]. This is characteristic for the cells under the influence of physiological and pathological conditions through the qualitative transformation in the CSK structure. This transformation occurs as the sequence of critical events due to the path of critical points $\delta_{*}, \delta_{c}$ in the attractor areas with corresponding self-similar solutions and the attractor phase spaces. The breathers and auto-solitary modes are responsible for the cell plasticity, which indicates mechanobiological properties reflecting the natural CSK transformation and the transition to the third attractor with the phase space related to the blow-up collective modes providing the cell differentiation dynamics. This scenario of collective modes transformation reflects the duality of the open complex dynamics in the ductile-brittle CSK cycle.

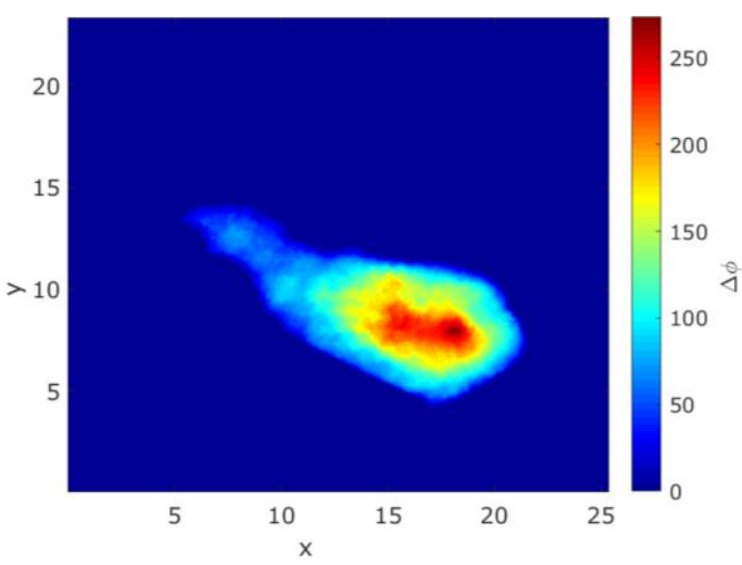

a)

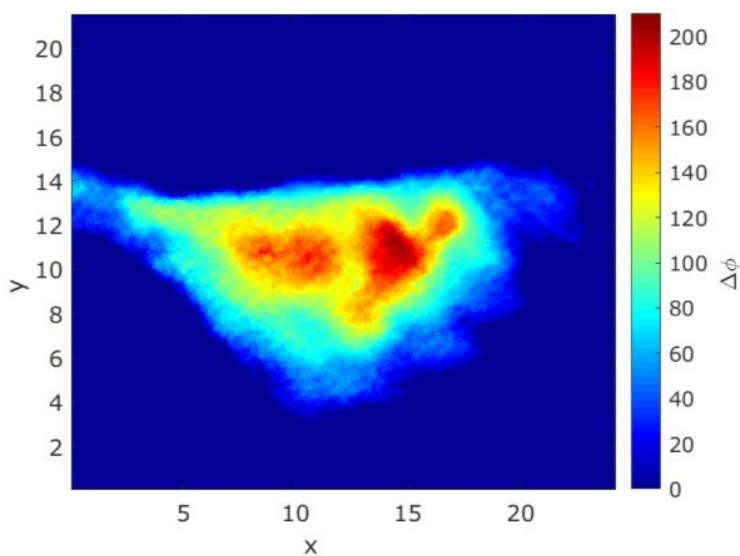

c)

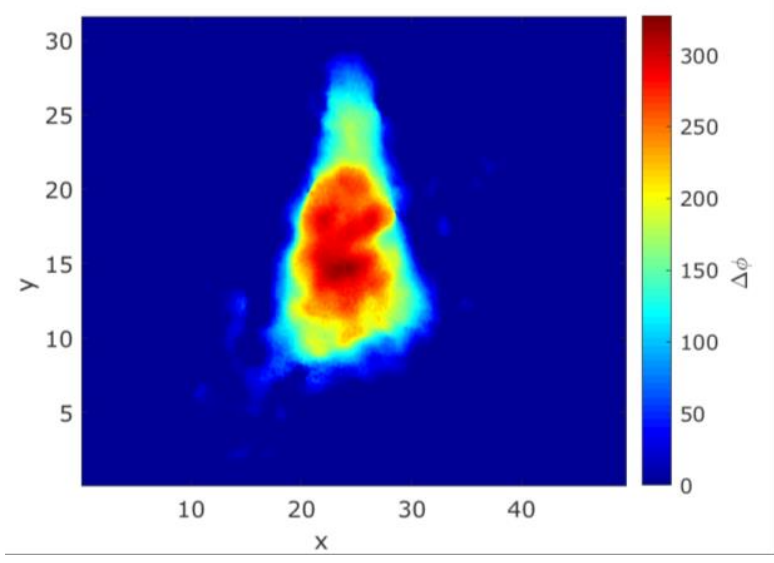

b)

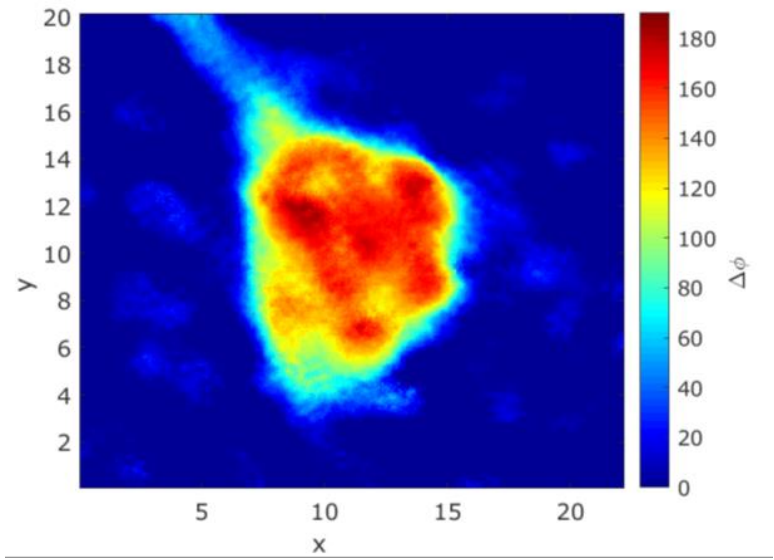

d)

Fig. 5. Phase images of the cells: a)-MCF-7, b)-HEK 293, c)-MCF-7, d)-HCT116. Units of measurements: $x, y-\mathrm{mkm} ; \Delta \phi-\mathrm{nm}$.

The structural-scaling transition events occur in the presence of the $\delta$ kinetics as the spinodal decomposition of the metastable free energy release caused by the dynamics of the open complex parameter. The qualitative different robust statistics (the lognormal and the power statistics), is characteristic for ductile and brittle behaviors can be used for the identification of the normal and cancerous CSK states [59]. The CSK cycle of the normal cells is associated with spatial-temporal distributed collective modes of open complexes, which follow to the multifractal dynamics associated with mentioned attractor types and the lognormal statistics of finite amplitude open complex fluctuations. This stochastic multiplicativity of open complex 
dynamics from the breathers to auto-solitary and blow-up modes provides the evolutionary "ductile" stability of the normal cells up to the stage of the cell division. The qualitative different scenario is observed for the cancer cells with pronounced "brittle" dynamics leading to the power law statistics and anomalous cell fragility for the arbitrary constraint [60]. The cancer cell behavior can be linked to the activity of the third attractor $\delta \rightarrow \delta_{c}$ under the initiation of the convergent multiscale "blow-up" dissipative structures subordinated entirely the CSK dynamics.

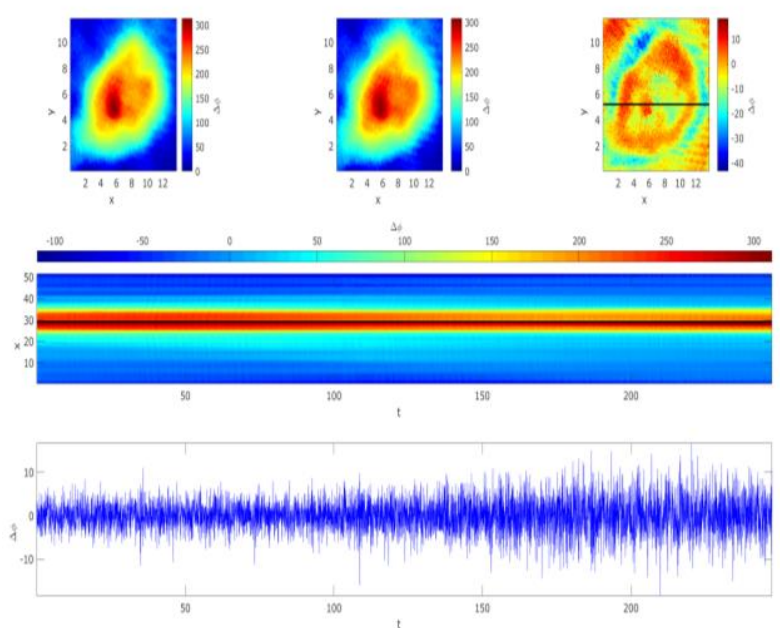

Fig. 6. Typical phase images registered at different time, difference frame, track diagram and 1D signal of the normal human breast epithelial cell MCF-10A. Units of measurements: $x, y-\mathrm{mkm} ; t-\mathrm{s} ; \Delta \phi-\mathrm{nm}$.

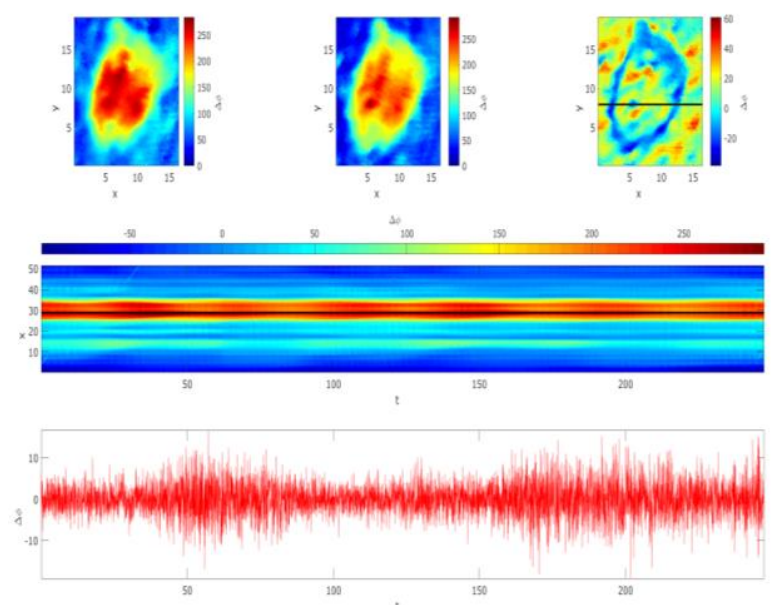

Fig. 7. Typical phase images registered at different time, difference frame, track diagram and 1D signal of the cancer human breast epithelial cell MCF-7. Units of measurements: $x, y-\mathrm{mkm} ; t-\mathrm{s} ; \Delta \phi-\mathrm{nm}$.

The study of the cell dynamics as the optical thickness fluctuations were conducted at the Perm Federal Research Center of the Urals Branch of the Russian Academy of Sciences using the MIM-340 laser interference microscope. Study of the nonlinear CSK dynamics using the data of the Laser Interference Microscopy (LIM) is the impact opportunity for the objectification of the cell states and cytological cancer diagnosis analyzing the time series of phase thickness fluctuations (in the "cross-sections" of the nucleus, the nucleolus, cytoplasm). The main advantages of the MIM-340 laser microscope are high resolution in the lateral plane $(10-100 \mathrm{~nm})$ and vertical $(0.3 \mathrm{~nm})$, the frequency recording of phase images $(33 \mathrm{~Hz})$ and the presence of an object table that allows the positioning the object (positioning accuracy is 150 $\mathrm{nm}$ ) [61]. The results of typical measurements are presented in Fig. 5 [62-64]. In these pictures, the adhesion region of living cells (green color), a thin layer of cytoplasm (yellow color), nuclei 
(red color) and nucleolus (dark red color) are clearly visualized. High spatial and temporal resolution of the LIM pattern allowed the analysis of dynamic processes in living cells using LIM track diagrams in different cell cross-sections (Fig. 6, 7). Optically dense area corresponds to the cell nuclei. These data were obtained by laser interference microscopy on 450 noncancerous and cancer cells.

Wavelet transform maximum modulus method (WTMM) [65-67] was used to analyze the LIM data and to establish the correlation between the finite amplitude temporal fluctuation of the phase thickness (associated with the cell thickness in the nucleus cross-sections) and qualitative different CSK dynamics providing the cell plasticity and fragility. 1D realization of the WTMM method was used to process the LIM data, that allowed one to get the singularity spectrum $f(\alpha)$ corresponding to the mono- and multifractal dynamics (Fig. 8) [62, 63]. Both scenarios display fat-tail distributions (log-normal and power laws) that are characteristic for the critical systems dynamics subject to the collective modes providing the spinodal decomposition of free energy metastability (free energy release) [68]. The lognormal multiplicative statistics reflects the influence on the CSK dynamics the mentioned types of collective modes in the course of the normal cell evolution as the structural-scaling transition in the open complex ensemble. The power law statistics reflects the cell fragility that is the consequence of pathological structural changes (corresponding to the range of structuralscaling parameter $\delta<\delta_{\mathrm{c}}$ ) leading to the subjection of the CSK dynamics to multiscale blow-up modes. These results allow the conclusion concerning the duality of multiscale open complex dynamics responsible for the evolutionary cell transformation as the ductile structural-scaling transition in the cell division and the brittle dynamics leading to the cell fragility and the cancer development.

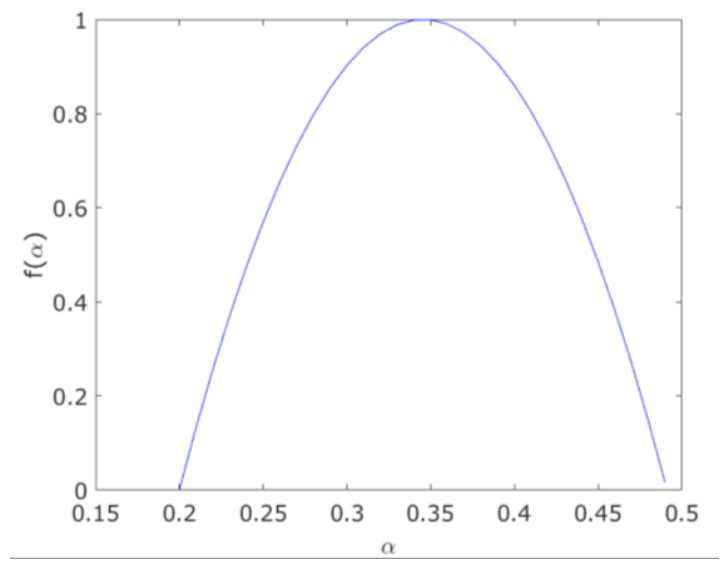

(a)

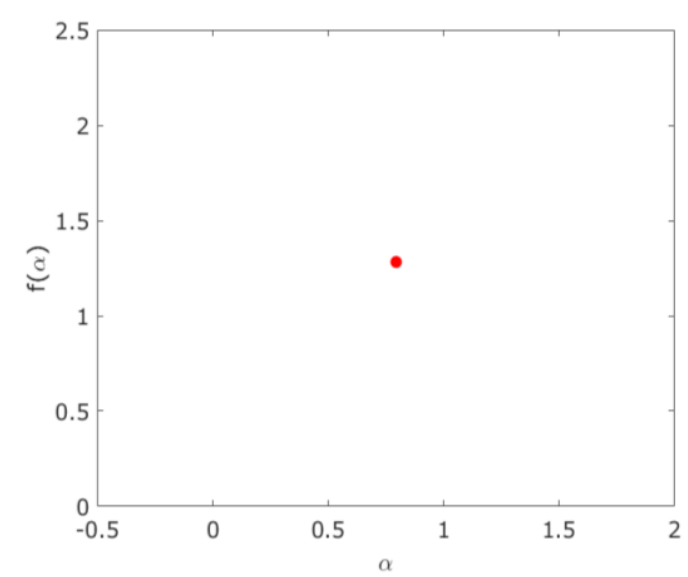

(b)

Fig. 8. Typical multifractal spectra of LIM data: "blue" is the normal breast cell, "red" is the cancer breast cell (carcinoma).

\section{DISCUSSION}

The mechanobiology of living cells is associated with the cytoskeleton (CSK) dynamics revealing the fundamental property of the cells qualified as the cell plasticity and the cell damage [69]. Plasticity is the phenomenon inherently linked to the collective behavior of mesodefects in the "biological crystals" and provides the unique mechanism of the defects induced momentum transfer and the structural memory as the expression scenario. The cell plasticity can be considered as the leading mechanism providing the vital CSK properties, including the DNA transformation, expression dynamics, and the cell division. Open complex mechanisms of plasticity and fragility are analyzed as specific type of critical phenomena in condensed matter with mesodefects - the structural-scaling transition. The expression dynamics, the self-organization of DNA and the cells are linked to the collective modes of the 
open complexes (breathers, auto-solitary waves, blow-up dissipative structures) responsible for the configuration mobility of the CSK structure and the cell division. The gene expression and the cell division being the vitality ground have natural links to the defects behavior and provide the evolutionary meaningful mechanisms of self-organization for normal cell dynamics or pathological scenario of defects induced fragility as the cancer precursor.

Development of collective open complex modes (breathers, auto-solitary, and blow-up) is the consequence of the local instability due to the subordination of the cell dynamics to the set of the self-similar solutions. Singular nature of the open complex as mesodefects allows one to link the dynamics of open complex collective modes with the dramatic change in the symmetry properties and the transformation pattern. The first symmetry breaking occurs in the course of mutual excitation of the breathers as the expression template related to the epigenetic landscape. The scale renormalization in the breather ensemble provides the long range correlation in more coarser epigenetic landscape, the second symmetry breaking due to the metastabilty decomposition in the range $\delta_{*}>\delta>\delta_{c}$ with the generation of auto-solitary modes and realization of the transcriptional dynamics, the histone topology and the histone package. The rescaling related to the auto-solitary modes leads to the second bifurcation at $\delta=\delta_{c}$ and the generation of blow-up open complex modes providing the cell division scenario. This pathway of the normal cell evolution occurs in the presence of three attractors excited consequently due to the structural-scaling transition and the convergence of trajectories related to the open complex dynamics of mentioned collective modes. The multiply "resonance" excitation of the third blow-up attractor is characteristic for the cancer cells revealing the divergent dynamics and the pronounced cell fragility. The resonance excitation of the multiply blow-up modes is typical for the epigenetic landscape with pronounced coarsening of the metastable potential in the presence of the infinite second minima. The mutual resonance blow-up kinetics of the open complexes leads to the spontaneous DNA fragmentation and low viscosity that is observed for the strongly coupled systems. The low viscosity could provide the anomalous proliferation as the metastasis mechanism.

Experimental study of the nonlinear CSK dynamics was conducted analyzing the time series of phase thickness fluctuations after the Laser Interference Microscopy in different cell "crosssections". The application of the WTMM method allowed the demonstration of the links of temporal correlations of finite-amplitude phase thickness fluctuation, dynamics of collective modes of open complexes and qualitative different CSK dynamics that are characteristic for the cell plasticity and fragility. The phase thickness fluctuations display the fat-tail distributions, the log-normal and the power laws, with multi- and monofractal singularity spectrum. The multifractal singularity spectrum in the case of the cell plasticity reflects the temporal sequences of the phase thickness fluctuation in the presence of mentioned open complex "singular" collective modes (breathers, auto-solitary, blow-up). The monofractal singularity spectrum and the power law of the phase thickness fluctuation are the consequence of the shifting of the CSK dynamics into the area of the attractor with the blow-up open complex dynamics that leads to the CSK fragmentation due to pronounced cell fragility. The open complex dynamics, which follows to the structural-scaling transition, allows the interpretation of the normal and cancer cell evolution scenario. The structural CSK susceptibility to both scenario is given by the values of structural-scaling parameter characterizing the nonlinearity (metastability) of the epigenetic landscape and corresponding open complex kinetics. The pathological changes of the CSK structure in the presence of the "monofractal" blow-up open complex dynamics leads to the cancer progression.

\section{ACKNOWLEDGMENTS}

Authors thank Pavel Ignatiev for unvalued support in the living cell experimental study and Prof. Mariano Bizzarri and Prof. Alessandro Giuliani for fruitful discussions. The work was 
financially supported by the Russian Federation via the Ministry of Science and Higher Education of the Russian Federation (project identifier RFMEFI60718X0202).

\section{REFERENCES}

1. Hoffman B.D., Crocker J.C. Cell mechanics: dissecting the physical responses of cells to force. Annual Review of Biomedical Engineering. 2009. V. 11. P. 259-288.

2. Gardel M.L., Kasza K.E., Brangwynne C.P., Liu J., Weitz D.A. Mechanical response of cytoskeletal networks. Methods in Cell Biology. 2008. V. 89. P. 487-519. doi: 10.1016/S0091-679X(08)00619-5.

3. Huber F., Schnauß J., Rönicke S., Rauch P., Müller K., Fütterer C., Käs J. Emergent complexity of the cytoskeleton: from single filaments to tissue. Advances in Physics. 2013. V. 62. № 1. P. 1-112.

4. Fletcher D.A., Mullins R.D. Cell mechanics and the cytoskeleton. Nature. 2010. V. 463. № 7280. P. 485-492.

5. Kauffman S.A. Metabolic stability and epigenesis in randomly constructed genetic nets. Journal of Theoretical Biology. 1969. V. 22. № 3. P. 437-467.

6. Huang S., Kauffman S.A. In: Complex gene regulatory networks-from structure to biological observables: cell fate determination. 2009. P. 1180-1213.

7. Huang S. Non-genetic heterogeneity of cells in development: more than just noise. Development. 2009. V. 136. № 23. P. 3853-3862.

8. Wang J., Xu L., Wang E., Huang S. The potential landscape of genetic circuits imposes the arrow of time in stem cell differentiation. Biophysical Journal. 2010. V. 99. № 1. P. 29-39.

9. Huang S., Eichler G., Bar-Yam Y., Ingber D.E. Cell fates as high-dimensional attractor states of a complex gene regulatory network. Physical Review Letters. 2005. V. 94. № 12. P. 128701.

10. Huang S. The molecular and mathematical basis of Waddington's epigenetic landscape: A framework for post-Darwinian biology? Bioessays. 2012. V. 34. № 2. P. 149-157.

11. Fumarola L., Urani C., Crosta G.F. Quantitative kinetics of damage and recovery of cytoskeletal structure by means of image analysis. Toxicology in vitro. 2005 . V. 19. № 7. P. 935-941.

12. Ingber D.E. Mechanical control of tissue growth: function follows form. Proceedings of the National Academy of Sciences. 2005. V. 102. № 33. P. 11571-11572.

13. Ingber D.E. Tensegrity-based mechanosensing from macro to micro. Progress in Biophysics and Molecular Biology. 2008. V. 97. № 2-3. P. 163-179.

14. Bonakdar N., Gerum R., Kuhn M., Spörrer M., Lippert A., Schneider W., Fabry B. Mechanical plasticity of cells. Nature Materials. 2016. V. 15. № 10. P. 1090-1094.

15. Naimark O.B. Defect-induced transitions as mechanisms of plasticity and failure in multifield continua. In: Advances in Multifield Theories for Continua with Substructure. Birkhäuser, Boston, MA, 2004. P. 75-114.

16. Naimark O.B. Structural-scaling transitions and localized distortion modes in the DNA double helix. Physical Mesomechanics. 2007. V. 1. № 10. P. 33-45.

17. Bizzarri M., Palombo A., Cucina A. Theoretical aspects of systems biology. Progress in Biophysics and Molecular Biology. 2013. V. 112. № 1-2. P. 33-43.

18. Naimark O. Nonlinear dynamics and damage induced properties of soft matter with application in oncology. AIP Conference Proceedings. 2017. V. 1882. № 1. P. 020052.

19. Peyrard M. Nonlinear dynamics and statistical physics of DNA. Nonlinearity. 2004. V. 17. № 2. P. R1.

20. Peyrard M., Bishop A.R. Statistical mechanics of a nonlinear model for DNA denaturation. Physical Review Letters. 1989. V. 62. № 23. P. 2755. 
21. Shigaev A.S., Ponomarev O.A., Lakhno V.D. Theoretical and Experimental Investigations of DNA Open States. Math. Biol. Bioinf. 2013. V. 8. № 2. P. 553-664. doi: $10.17537 / 2013.8 .553$.

22. Wartell R.M., Benight A.S. Thermal denaturation of DNA molecules: a comparison of theory with experiment. Physics Reports. 1985. V. 126. № 2. P. 67-107.

23. Likhachev I.V., Lakhno V.D. The direct investigation of DNA denaturation in PeyrardBishop-Dauxois model by molecular dynamics method. Chemical Physics Letters. 2019. V. 727. P. 55-58.

24. Likhachev I.V., Lakhno V.D. Investigation of DNA denaturation in Peyrard-BishopDauxois model by molecular dynamics method. The European Physical Journal B. 2019. V. 92. № 11. P. 253.

25. Nikitiuk A.S., Korznikova E.A., Dmitriev S.V., Naimark O.B. DNA breathers and cell dynamics. Mathematical Biology and Bioinformatics. 2019. V. 14. № 1. P. 137-149. doi: $10.17537 / 2019.14 .137$

26. Nikitiuk A.S., Korznikova E.A., Dmitriev S.V., Naimark O.B. Nonlinear dynamics of DNA with topological constraints. Letters on Materials. 2018. V. 8. № 4. P. 489-493.

27. Tsuchiya M., Giuliani A., Yoshikawa K. Single-Cell Reprogramming in Mouse Embryo Development through a Critical Transition State. Entropy. 2017. V. 19. № 11. P. 584.

28. Woese C.R. A new biology for a new century. Microbiology and Molecular Biology Reviews. 2004. V. 68. № 2. P. 173-186.

29. Leontovich M. Introduction to Thermodynamics. Statistical Physics. Moscow: High School, 1983. $416 \mathrm{p}$.

30. Goldenfeld N., Woese C. Biology's next revolution. Nature. 2007. V. 445. № 7126. P. 369-369.

31. Goldenfeld N., Woese C. Life is physics: evolution as a collective phenomenon far from equilibrium. Annu. Rev. Condens. Matter Phys. 2011. V. 2. № 1. P. 375-399.

32. Waddington C.H. Canalization of development and the inheritance of acquired characters. Nature. 1942. V. 150. № 3811. P. 563-565.

33. Waddington C.H. The strategy of the genes. Routledge, 2014.

34. Goldberg A.D., Allis C.D., Bernstein E. Epigenetics: a landscape takes shape. Cell. 2007. V. 128. № 4. P. 635-638.

35. Tsuchiya M., Selvarajoo K., Piras V., Tomita M., Giuliani A. Local and global responses in complex gene regulation networks. Physica A: Statistical Mechanics and its Applications. 2009. V. 388. № 8. P. 1738-1746.

36. Tsuchiya M., Hashimoto M., Takenaka Y., Motoike I.N., Yoshikawa K. Global genetic response in a cancer cell: Self-organized coherent expression dynamics. PLoS One. 2014. V. 9. № 5. Article No. e97411.

37. Aldana M., Balleza E., Kauffman S., Resendiz O. Robustness and evolvability in genetic regulatory networks. Journal of Theoretical Biology. 2007. V. 245. № 3. P. 433-448.

38. Kurdyumov S.P. Evolution and self-organization laws in complex systems. Advances in Theoretical Physics. 1990. P. 134.

39. Naimark O.B. Structural-scale transitions in solids with defects and symmetry aspects of field theory. Physical Mesomechanics. 2010. V. 13. № 5-6. P. 306-317.

40. Damasco A., Giuliani A. A resonance based model of biological evolution. Physica A: Statistical Mechanics and its Applications. 2017. V. 471. P. 750-756.

41. Naimark O. Mesoscopic cell dynamics in different environment and problem of cancer. AIP Conference Proceedings. 2019. V. 2167. № 1. P. 020237.

42. Longo G., Montévil M. From physics to biology by extending criticality and symmetry breakings. In: Perspectives on Organisms. Berlin, Heidelberg: Springer, 2014. P. 161185.

43. Longo G., Montévil M.R., Pocheville A. From bottom-up approaches to levels of organization and extended critical transitions. Frontiers in Physiology. 2012. V. 3. P. 232. 
44. Huang S., Ingber D.E. Shape-dependent control of cell growth, differentiation, and apoptosis: switching between attractors in cell regulatory networks. Experimental Cell Research. 2000. V. 261. № 1. P. 91-103.

45. Huang S., Ingber D.E. A non-genetic basis for cancer progression and metastasis: selforganizing attractors in cell regulatory networks. Breast Disease. 2007. V. 26. № 1. P. 27 54.

46. Auffray C., Nottale L. Scale relativity theory and integrative systems biology: 1: founding principles and scale laws. Progress in Biophysics and Molecular Biology. 2008. V. 97. № 1. P. 79-114.

47. Auffray C., Imbeaud S., Roux-Rouquié M., Hood L. Self-organized living systems: conjunction of a stable organization with chaotic fluctuations in biological space-time. Philosophical Transactions of the Royal Society of London. Series A: Mathematical, Physical and Engineering Sciences. 2003. V. 361. № 1807. P. 1125-1139.

48. Bailly F., Longo G. Extended critical situations: the physical singularity of life phenomena. Journal of Biological Systems. 2008. V. 16. № 02. P. 309-336.

49. Bailly F., Longo G. Biological organization and anti-entropy. Journal of Biological Systems. 2009. V. 17. № 01. P. 63-96.

50. Landau L.D. On the theory of phase transitions. Zh. Eksp. Teor. Fiz. 1937. V. 11. P. 19.

51. Ginzburg V.L., Landau L.D. On the theory of superconductors. Zh. Eksp. Teor. Fiz. 1950. V. 20. P. 1064-1082.

52. Weinberg A.M. On the Relation Between Information and Energy Systems. A Family of Maxwell's Demons. Interdisciplinary Science Reviews. 1982. V. 7. № 1. P. 47-52.

53. Tsuchiya M., Giuliani A., Hashimoto M., Erenpreisa J., Yoshikawa K. Emergent SelfOrganized Criticality in gene expression dynamics: Temporal development of global phase transition revealed in a cancer cell line. PLoS One. 2015. V. 10. № 6. Article No. e0128565.

54. Tsuchyia M., Wong S.T., Yeo Z.X., Colosimo A., Palumbo M.C., Farina L., Selvarajoo K. Gene expression waves: cell cycle independent collective dynamics in cultured cells. The FEBS Journal. 2007. V. 274. № 11. P. 2878-2886.

55. Naimark O.B. Collective properties of defect ensembles and some nonlinear problems of plasticity and fracture. Physical Mesomechanics. 2003. V. 6. № 4. P. 39-64.

56. Naimark O.B., Nikitiuk A.S., Baudement M.O., Forné T., Lesne A. The physics of cancer: The role of epigenetics and chromosome conformation in cancer progression. AIP Conference Proceedings. 2016. V. 1760. № 1. P. 020051.

57. Barenblatt G.I., Zel'dovich Y.B. Intermediate asymptotics in mathematical physics. Russian Math. Surveys. 1971. V. 26. № 2. P. 45-61.

58. Barenblatt G.I. Similarity, Self-Similarity, Intermediate Asymptotics. NY: Springer US, 1979. $218 \mathrm{p}$.

59. Naimark O.B. Some regularities of scaling in plasticity, fracture, and turbulence. Physical Mesomechanics. 2016. V. 19. № 3. P. 307-318.

60. Naimark O.B., Uvarov S.V., Davydova M.M., Bannikova I.A. Multiscale statistical laws of dynamic fragmentation. Physical Mesomechanics. 2017. V. 20. № 1. P. 90-101.

61. Ignatyev P.S., Indukaev K.V., Osipov P.A., Sergeev I.K. Laser interference microscopy for nanobiotechnologies. Biomedical Engineering. 2013. V. 47. № 1. P. 32-35.

62. Naimark O. Nonlinear dynamics and damage induced properties of soft matter with application in oncology. AIP Conference Proceedings. 2017. V. 1882. № 1. P. 020052.

63. Naimark O. Mesoscopic cell dynamics in different environment and problem of cancer. AIP Conference Proceedings. 2019. V. 2167. № 1. P. 020237.

64. Naimark O.B., Grishko V.V., Bayandin Yu.V., Nikityuk A.S. Mechanobiological study of the dynamics and morphology of cell structures by laser microscopy and applications in oncology. Perm Federal Research Center Journal. 2020. V. 1. P. 70-87. 
65. Gerasimova E., Audit B., Roux S.G., Khalil A., Argoul F., Naimark O., Arneodo A. Multifractal analysis of dynamic infrared imaging of breast cancer. Europhysics Letters. 2014. V. 104. № 6. P. 68001.

66. Gerasimova E., Audit B., Roux S.G., Khalil A., Gileva O., Argoul F., Naimark O., Arneodo A. Wavelet-based multifractal analysis of dynamic infrared thermograms to assist in early breast cancer diagnosis. Frontiers in Physiology. 2014. V. 5. P. 176. doi: $10.3389 /$ fphys.2014.00176.

67. Gerasimova-Chechkina E., Toner B., Marin Z., Audit B., Roux S.G., Argoul F., Khalil A., Gileva O., Naimark O., Arneodo A. Comparative multifractal analysis of dynamic infrared thermograms and X-ray mammograms enlightens changes in the environment of malignant tumors. Frontiers in Physiology. 2016. V. 7. P. 336.

68. Naimark O.B. Energy release rate and criticality of multiscale defects kinetics. International Journal of Fracture. 2016. V. 202. № 2. P. 271-279.

69. Bizzarri M., Naimark O., Nieto-Villa J., Fedeli V., Giuliani A. Complexity in Biological Organization: Deconstruction (and Subsequent Restating) of Key Concepts. Entropy. 2020. V. 22. № 8. P. 885. doi: 10.3390/e22080885.

Received 19.08.2020. Revised 05.10.2020. Published 11.11.2020. 